\title{
UNIVERSAL SPECTRA, UNIVERSAL TILING SETS AND THE SPECTRAL SET CONJECTURE
}

\author{
STEEN PEDERSEN and YANG WANG
}

\begin{abstract}
A subset $\Omega$ of $\mathrm{R}^{d}$ with finite positive Lebesgue measure is called a spectral set if there exists a subset $\Lambda \subset$ R such that $\mathscr{E}_{\Lambda}:=\left\{e^{i 2 \pi\langle\lambda, x\rangle}: \lambda \in \Lambda\right\}$ form an orthogonal basis of $L^{2}(\Omega)$. The set $\Lambda$ is called a spectrum of the set $\Omega$. The Spectral Set Conjecture states that $\Omega$ is a spectral set if and only if $\Omega$ tiles $\mathrm{R}^{d}$ by translation. In this paper we prove the Spectral Set Conjecture for a class of sets $\Omega \subset$ R. Specifically we show that a spectral set possessing a spectrum that is a strongly periodic set must tile $\mathrm{R}$ by translates of a strongly periodic set depending only on the spectrum, and vice versa.
\end{abstract}

\section{Introduction}

Let $\Omega$ be a (Lebesgue) measurable subset of $\mathrm{R}$ with finite positive measure. For $t \in \mathrm{R}$ let $\Omega+t:=\{x+t: x \in \Omega\}$ denote the translate of $\Omega$ by $t$. We say that $\Omega$ tiles $\mathrm{R}$ by translation if there exists a subset $\mathscr{T} \subset \mathrm{R}$ so that $\mathrm{R} \backslash \bigcup_{t \in \mathscr{T}}(\Omega+t)$ is a set of measure zero and $(\Omega+t) \cap\left(\Omega+t^{\prime}\right)$ is a set of measure zero whenever $t, t^{\prime} \in \mathscr{T}$ are distinct. In the affirmative case $\mathscr{T}$ is called a tiling set for $\Omega$, and $(\Omega, \mathscr{T})$ is called a tiling pair. Similarly, we say that $\Omega$ tiles the non-negative half line $\mathrm{R}^{+}=[0, \infty)$ if there exists a subset $\mathscr{T} \subset \mathrm{R}$ such that $\mathrm{R}^{+} \backslash \bigcup_{t \in \mathscr{T}}(\Omega+t)$ is a set of measure zero and $(\Omega+t) \cap\left(\Omega+t^{\prime}\right)$ is a set of measure zero whenever $t, t^{\prime} \in \mathscr{T}$ are distinct. Sets that tile the real line by translation have been studied recently, e.g., [9], [8], [7].

For $\lambda \in R$ we introduce the functions

$$
e_{\lambda}(x):=e^{i 2 \pi \lambda x}, \quad x \in \mathrm{R} .
$$

We say that $\Omega$ is a spectral set if there exists a subset $\Lambda \subset R$ so that the functions $\mathscr{E}_{\Lambda}:=\left\{e_{\lambda}: \lambda \in \Lambda\right\}$ form an orthogonal basis for $L^{2}(\Omega)$, the Hilbert space of complex valued square integrable functions on $\Omega$ with the inner product

$$
\langle f, g\rangle:=\int_{\Omega} \overline{f(x)} g(x) d x .
$$

Received August 25, 1998. 
If the functions in $\mathscr{E}_{\Lambda}$ form an orthogonal basis for $L^{2}(\Omega)$, then we call $(\Omega, \Lambda)$ a spectral pair and $\Lambda$ a spectrum for $\Omega$. Spectral sets have recently been studied in various contexts, e.g., [3], [4], [5], [10], [8], [6].

One of the main open questions concerning spectral sets is the following conjecture, first proposed by Fuglede [3]:

Spectral Set Conjecture. Let $\Omega$ be a measurable subset of $\mathrm{R}^{d}$ with finite positive Lebesgue measure. Then $\Omega$ is a spectral set if and only if $\Omega$ tiles $\mathrm{R}^{d}$ by translation.

In this paper we study the one dimensional case of the Spectral Set Conjecture. A special class of sets we study consists of tiles that tile the non-negative half line $\mathrm{R}^{+}$by translation. We prove:

THEOREM 1.1. Let $\Omega$ be a subset of $\mathrm{R}$ with finite positive Lebesgue measure. Suppose that $\Omega$ tiles $\mathrm{R}^{+}$by translation. Then $\Omega$ tiles $\mathrm{R}$ by translation and is a spectral set.

Let $N:=\{1,2,3, \ldots\}$ be the set of natural numbers and $Z^{+}:=\{0,1,2, \ldots\}$ be the set of non-negative integers. For any $n \in \mathrm{N}$ let $\mathrm{Z}_{n}^{+}:=\{0,1, \ldots, n-1\}$. For any $A, B \subseteq \mathrm{Z}$ we write

$$
A+B:=\{a+b: a \in A, b \in B\}
$$

for the Minkowski sum of $A$ and $B$. We will write $A \oplus B$ if each element in $A+B$ has a unique decomposition of the form $a+b$ with $a \in A$ and $b \in B$.

DEFINITION 1.2. We call $A \subset \mathrm{Z}^{+}$a direct summand of $\mathrm{Z}_{n}^{+}$if there exists a $B \subset \mathrm{Z}^{+}$such that $A \oplus B=\mathrm{Z}_{n}^{+}$. We call a subset $\mathscr{T}$ of $\mathrm{R}$ a strongly periodic set if there exist an $n \in \mathrm{N}$ and a direct summand $A \subset \mathrm{Z}^{+}$of $\mathrm{Z}_{n}^{+}$such that $\mathscr{T}=\alpha(A \oplus n Z)$ for some non-zero $\alpha \in \mathrm{R}$.

In [8] it was shown that certain tiles that tile $\mathrm{R}$ by translation are spectral sets that possess the so-called universal spectra, in the sense that the spectra depend only on the tiling sets, not the tiles. Our main theorem below strengthens this notion by providing a large new class of tiles that possess universal spectra. It shows that a tile that tiles $R$ by the translates of a strongly periodic set must have a universal spectrum that is also a strongly periodic set. More importantly, the theorem also gives rise to the notion of universal tiling set, which can be viewed as the dual of universal spectrum. We show that a spectral set that possesses a spectrum that is a strongly periodic set must have a universal tiling set depending only on the spectrum.

THEOREM 1.3. Let $\Omega$ be a subset of $\mathrm{R}$ with finite positive measure. Suppose that there exists a strongly periodic set $\Lambda \subset \mathrm{R}$ such that $(\Omega, \Lambda)$ is a spectral 
pair. Then there exists a strongly periodic set $\mathscr{T} \subset \mathrm{R}$ depending only on $\Lambda$ such that $\Omega$ tiles $\mathrm{R}$ by translates of $\mathscr{T}$. Conversely, suppose that there exists a strongly periodic set $\mathscr{T} \subset \mathrm{R}$ such that $\Omega$ tiles $\mathrm{R}$ by translates of $\mathscr{T}$. Then there exists a strongly periodic set $\Lambda \subset \mathrm{R}$ depending only on $\mathscr{T}$ such that $(\Omega, \Lambda)$ is a spectral pair.

The strongly periodic sets $\Lambda$ and $\mathscr{T}$ in Theorem 1.3 are duals of each other, and for each given one the other is constructed explicitly in $\S 4$. In fact we prove a stronger version of Theorem 1.3 there. For the rest of the paper, in $\$ 2$ we state a result on the structure of strongly periodic sets, first shown in [2]. In $\S 3$ we classify tiles that tile $\mathrm{R}^{+}$by translation. The classification is used to prove Theorem 1.1.

\section{Structure of Strongly Periodic Sets}

In this section we classify subsets $A, B$ of $Z^{+}$satisfying $A \oplus B=Z_{n}^{+}$for some $n \in \mathrm{N}$. The classification is based on a theorem of de Bruijn [2] establishing the structure of subsets of $\mathrm{Z}^{+}$that tile $\mathrm{Z}^{+}$by translation. To formulate the result we first introduce some notation regarding divisibility. For $r, s \in \mathrm{Z}$ we use $r \mid s$ to mean that $r$ divides $s$; for $r \in \mathrm{Z}$ and $A \subseteq \mathrm{Z}$ we use $r \mid A$ to mean that $r$ divides every $a \in A$.

Proposition 2.1 (de Bruijn). Let $A, B \subseteq \mathrm{Z}^{+}$such that $A \oplus B=\mathrm{Z}^{+}$and $A \neq Z^{+}, B \neq Z^{+}$. Then there exists an integer $r>1$ such that $r \mid A$ or $r \mid B$. Furthermore, if $r \mid B$ and $B=r \widetilde{B}$ then there exists an $\widetilde{A} \subseteq \mathrm{Z}^{+}$such that

$$
A=\mathrm{Z}_{r}^{+} \oplus r \widetilde{A}, \text { and } \tilde{A} \oplus \widetilde{B}=\mathrm{Z}^{+} .
$$

Proof. A proof can be found in de Bruijn [2]. For the sake of self-containment we give a short proof here.

Without loss of generality we assume $1 \in A$. Let $r$ be the smallest non-zero member of $B$. For each $m \in \mathrm{N}$ let $A_{m} \subseteq A$ and $B_{m} \subseteq B$ be the minimal subsets so that

$$
\mathrm{Z}_{m r}^{+} \subseteq A_{m}+B_{m} .
$$

It follows immediately from the minimality and the uniqueness in $A \oplus B$ that

$$
A_{m}=A \cap \mathrm{Z}_{m r}^{+}, \quad B_{m}=B \cap \mathrm{Z}_{m r}^{+} .
$$

Observe that $\mathrm{Z}_{(m+1) r}^{+} \backslash \mathrm{Z}_{m r}^{+}=\mathrm{Z}_{r}^{+}+m r$. So

$$
A_{m+1} \backslash A_{m} \subseteq \mathrm{Z}_{r}^{+}+m r, \quad B_{m+1} \backslash B_{m} \subseteq \mathrm{Z}_{r}^{+}+m r .
$$


We show by induction on $m$ that there are subsets $C_{m}$ and $D_{m}$ of $Z^{+}$such that

$$
A_{m}=\mathrm{Z}_{r}^{+}+r C_{m}, \quad B_{m}=r D_{m} .
$$

Let $C_{1}:=\{0\}$ and $D_{1}:=\{0\}$. Then $A_{1}=Z_{r}^{+}+r C_{1}$ and $B_{1}=r D_{1}$ as required. Suppose that $C_{m}, D_{m} \subseteq \mathrm{Z}^{+}$have been constructed so that $A_{m}=$ $\mathrm{Z}_{r}^{+}+r C_{m}$ and $B_{m}=r D_{m}$. If $\mathrm{Z}_{(m+1) r}^{+} \subseteq A_{m}+B_{m}$, then $A_{m+1}=A_{m}$ and $B_{m+1}=B_{m}$, and so it suffices to set $C_{m+1}:=C_{m}$ and $D_{m+1}:=D_{m}$ to complete the proof.

Now suppose that $\mathrm{Z}_{(m+1) r}^{+} \not A_{m}+B_{m}$. Let $j \in \mathrm{Z}_{r}^{+}$. If $j+m r \in A_{m}+B_{m}=$ $\mathrm{Z}_{r}^{+}+r\left(C_{m}+D_{m}\right)$ then $m \in C_{m}+D_{m}$ and therefore $\mathrm{Z}_{r}^{+}+m r \subseteq A_{m}+B_{m}$, contradicting $\mathrm{Z}_{(m+1) r}^{+} \nsubseteq A_{m}+B_{m}$. Hence,

$$
\left(Z_{r}^{+}+m r\right) \cap\left(A_{m}+B_{m}\right)=\emptyset .
$$

It follows that $m r \in A_{m+1}$ or $m r \in B_{m+1}$.

If $m r \in B_{m+1}$, then $A_{m+1}=A_{m}$ and $B_{m+1}=B_{m} \cup\{\mathrm{rm}\}$. Hence we may set $C_{m+1}:=C_{m}$ and $D_{m+1}:=D_{m} \cup\{m\}$.

Assume that $m r \in A_{m+1}$. Let $j \in \mathrm{Z}_{r}^{+}$. We have shown above that $j+$ $m r \notin A_{m}+B_{m}$, so $j+m r=a+b$ for $a \in A_{m+1} \backslash A_{m}, b \in B_{m+1}$ or $a \in A_{m}, b \in B_{m+1} \backslash B_{m}$. If $b \in B_{m+1} \backslash B_{m}$ then $(m+1) r-b \in Z_{r}^{+}$. Thus $m r+r=((m+1) r-b)+b$ constitute two different decompositions of the same element in $A \oplus B$, a contradiction. This yields $a \in A_{m+1} \backslash A_{m}$. If $b \neq 0$ then $B_{m}=r D_{m}$ and $B_{m+1} \backslash B_{m} \subseteq Z_{r}^{+}+m r$ implies that $b \geq r$. So $j+m r=a+b \geq m r+r>j+m r$, again a contradiction. So $b=0$ and therefore $j+m r=a \in A_{m+1}$. It follows that

$$
A_{m+1}=A_{m} \cup\left(Z_{r}^{+}+m r\right) .
$$

The inductions steps are now complete by setting $C_{m+1}:=C_{m} \cup\{m\}$ and $D_{m+1}:=D_{m}$.

Finally, the proposition follows by letting $\widetilde{A}:=\bigcup_{m=1}^{\infty} C_{m}$ and $\widetilde{B}=\bigcup_{m=1}^{\infty} D_{m}$.

Proposition 2.1 immediately leads to the following classification of strongly periodic sets.

Corollary 2.2. Let $A, B \subseteq Z^{+}$such that $A \oplus B=Z_{n}^{+}$and $A \neq Z_{n}^{+}$, $B \neq \mathrm{Z}_{n}^{+}$. Then there exists an $r>1$ such that $r \mid n$ and either $r \mid A$ or $r \mid B$. Furthermore, if $r \mid B$ and $B=r \widetilde{B}$ then there exists an $\widetilde{A} \subset Z^{+}$so that

$$
A=\mathrm{Z}_{r}^{+} \oplus r \widetilde{A}, \quad \text { and } \quad \widetilde{A} \oplus \widetilde{B}=\mathrm{Z}_{\frac{n}{r}}^{+} .
$$

Proof. Suppose that $1 \in A$. Applying Proposition 2.1 to $A \oplus\left(B \oplus n Z^{+}\right)=$ $\mathrm{Z}^{+}$yields an $r>1$ and a set $\widetilde{A}$ so that $A=\mathrm{Z}_{r}^{+} \oplus r \widetilde{A}$ and $r \mid\left(B \oplus n \mathrm{Z}^{+}\right)$. Since 
$0 \in B$ and $0 \in \mathrm{Z}^{+}$it follows that $r \mid n$ and $r \mid B$. Finally, $\mathrm{Z}_{r}^{+} \oplus r(\widetilde{A}+\widetilde{B})=$ $A \oplus B=\mathrm{Z}_{n}^{+}$implies $\widetilde{A} \oplus \widetilde{B}=\mathrm{Z}_{\frac{n}{r}}^{+}$.

Corollary 2.3. Let $A, B \subseteq Z^{+}$such that $A \oplus B=Z_{n}^{+}$. Assume that $1 \in A$. Then there exists a unique finite sequence $d_{0}=1, d_{1}, \ldots, d_{k-1}, d_{k}=n$ in $\mathrm{N}$ with $r_{j}:=d_{j} / d_{j-1} \in \mathrm{N}$ and $r_{j}>1$ for $1 \leq j \leq k$ such that

$$
\begin{aligned}
& A=d_{0} \mathrm{Z}_{r_{1}}^{+} \oplus d_{2} \mathrm{Z}_{r_{3}}^{+} \oplus \cdots, \\
& B=d_{1} \mathrm{Z}_{r_{2}}^{+} \oplus d_{3} \mathrm{Z}_{r_{4}}^{+} \oplus \cdots .
\end{aligned}
$$

Proof. Since $1 \in A$, the proof of Proposition 2.1 yields $A=\mathrm{Z}_{r_{1}}^{+} \oplus r_{1} \widetilde{A}$ and $B=r_{1} \widetilde{B}$ where $r_{1}=\min \{b: b \in B, b \neq 0\}$, and $\widetilde{A} \oplus \widetilde{B}=\mathrm{Z}_{\frac{n}{r_{1}}}^{+}$. The proof is completed by applying Corollary 2.2 iteratively to $\widetilde{A} \oplus \widetilde{B}=\mathrm{Z}_{\frac{r_{1}}{r_{1}}}^{+}$. Note that the uniqueness follows from the fact that $r_{1}=d_{1} / d_{0}=\min \left\{b: \stackrel{r_{1}}{b} \in B, b \neq 0\right\}$, $r_{2}=d_{2} / d_{1}=\{a: a \in \widetilde{A}, a \neq 0\}$, etc.

Corollary 2.4. Suppose that $A, B \subseteq \mathrm{Z}^{+}$such that $A \oplus B=\mathrm{Z}^{+}$, and that $B$ is finite. Then $B$ is a direct summand of $Z_{n}^{+}$for some $n \in \mathrm{N}$.

Proof. By the same argument for Corollary $2.3 B$ must have the form (2.1) or (2.2), depending on whether $1 \in B$. So $B$ must be a direct summand of $Z_{n}^{+}$ for some $n \in \mathrm{N}$.

Call a polynomial a $0-1$ polynomial if each of its coefficients is either 0 or 1 . We associate each finite $A \subseteq \mathrm{Z}^{+}$with the following $0-1$ polynomial

$$
A(x):=\sum_{a \in A} x^{a},
$$

called the characteristic polynomial of $A$. Clearly every $0-1$ polynomial is the characteristic polynomial of the set of exponents corresponding to its non-zero coefficients. If $A, B, C \subseteq \mathrm{Z}^{+}$are finite, then $A \oplus B=C$ if and only if $A(x) B(x)=C(x)$. We call a $0-1$ polynomial c-irreducible if $A(x) \neq$ $A_{1}(x) A_{2}(x)$ for any $0-1$ polynomials $A_{1}(x) \not \equiv 1, A_{2}(x) \not \equiv 1$. The following result was first stated in [1] (simple examples, however, show that Lemma 1 in [1] is false).

THEOREM 2.5. Let $n>1$. Then every factorization of $\frac{x^{n}-1}{x-1}$ into c-irreducible $0-1$ polynomials has the form

$$
\frac{x^{n}-1}{x-1}=F_{p_{1}}(x) F_{p_{2}}\left(x^{p_{1}}\right) F_{p_{3}}\left(x^{p_{1} p_{2}}\right) \ldots F_{p_{k}}\left(x^{p_{1} p_{2} \ldots p_{k-1}}\right),
$$


where $F_{m}(x):=\frac{x^{m}-1}{x-1}$, all $p_{j}$ are primes (not necessarily distinct) and $n=$ $p_{1} p_{2} \ldots p_{k}$.

Proof. This is a direct consequence of Corollary 2.3, by observing that

$$
\mathrm{Z}_{p_{1} p_{2} \cdots p_{k}}^{+}=\mathrm{Z}_{p_{1}}^{+} \oplus p_{1} \mathrm{Z}_{p_{2}}^{+} \oplus p_{1} \ldots p_{k-1} \mathrm{Z}_{p_{k}}
$$

Note that each term in the factorization is c-irreducible, because it contains a prime number of terms.

\section{Tiling the Non-Negative Real Line}

Let $\Omega \subset \mathrm{R}$ be a tile with finite and positive Lebesgue measure that tiles $\mathrm{R}^{+}$by translates of $\mathscr{T}$. In this case we will write $\Omega \oplus \mathscr{T}=\mathrm{R}^{+}$. In this section we derive the structure of tiles $\Omega \subset \mathrm{R}$ that tile $\mathrm{R}^{+}$by translation.

THEOREM 3.1. Let $\Omega \subset \mathrm{R}$ with finite positive Lebesgue measure. Suppose that $\Omega$ tiles $\mathrm{R}^{+}$by translation. Then there exists an affine map $\varphi(x)=a x+b$ such that

$$
\varphi(\Omega)=[0,1]+B
$$

for some finite subset $B \subset Z^{+}$with $0 \in B$. Furthermore, $B$ is a direct summand of $\mathrm{Z}_{n}^{+}$for some $n \in \mathrm{N}$. Hence $\Omega$ tiles $\mathrm{R}$ by translation.

Proof. In this proof, all set relations involving the tile $\Omega$ will be interpreted as up to measure zero sets.

Let $\mathscr{T} \subset \mathrm{R}$ such that $\Omega \oplus \mathscr{T}=\mathrm{R}^{+}$. We first examine the special case $\mathscr{T}=\left\{0,1, t_{2}, t_{3}, \ldots\right\}$ where $t_{j}>1$ for all $j \geq 2$. In this special case we prove that $\Omega=[0,1]+B$ for some $B \subset Z^{+}$and $0 \in B$. Let $\mathscr{T}_{n}=\mathscr{T} \cap[0, n-1]$ and $\Omega_{n}=\Omega \cap[0, n]$. We claim that $\mathscr{T}_{n} \subset \mathrm{Z}^{+}$and $\Omega_{n}=[0,1]+B_{n}$ for some $B_{n} \subset \mathrm{Z}^{+}$, by induction on $n$.

Since $t_{j}>1$, we must have $[0,1] \subseteq \Omega$. So the claim is clearly true for $n=1$. Assume that the claim is true for all $n<k$. We show that the claim is also true for $n=k$. We divide the proof into two cases: $\Omega_{k-1} \subsetneq \Omega_{k}$ and $\Omega_{k-1}=\Omega_{k}$. Suppose that $\Omega_{k-1} \subsetneq \Omega_{k}$. Then $\Omega \cap(k-1, k] \neq \emptyset$. If $\Omega_{k} \neq[0,1]+B_{k}$ for any $B_{k} \subset Z^{+}$, then $\Omega \cap(k-1, k] \subsetneq(k-1, k]$. Hence there exists a $t \in \mathscr{T}$ such that $(\Omega+t) \cap(k-1, k] \neq \emptyset$. Note that $t \in \mathscr{T}_{k-1}$, so $t \in \mathrm{Z}^{+}$. It follows that

$$
\emptyset \subsetneq \Omega \cap(k-1-t, k-t] \subsetneq(k-1-t, k-t],
$$

contradicting the inductive hypothesis. So $\Omega_{k}=[0,1]+B_{k}$ for some $B_{k} \subset \mathrm{Z}^{+}$. The assumption that $\Omega_{k-1} \subsetneq \Omega_{k}$ now implies that $B_{k}=B_{k-1} \cup\{k-1\}$, so $\mathscr{T}_{k}=\mathscr{T}_{k-1}$. This proves the claim for $n=k$ in the first case. Suppose that $\Omega_{k-1}=\Omega_{k}$. Then $\Omega_{k}=[0,1]+B_{k}$ with $B_{k}=B_{k-1}$. Therefore $\mathscr{T}_{k}=$ 
$\mathscr{T}_{k-1} \cup\{k-1\}$. This completes the induction steps and proves the claim. So we have shown that $B, \mathscr{T} \subseteq \mathrm{Z}^{+}$, and clearly $0 \in B$.

It remains to show that $B$ is a direct summand of $Z_{n}^{+}$for some $n \in \mathrm{N}$. Observe that $B \oplus \mathscr{T}=\mathrm{Z}^{+}$. Therefore $B$ is a direct summand of $Z_{n}^{+}$for some $n \in \mathrm{N}$ by Corollary 2.4.

In general, suppose that $\Omega$ tiles $\mathrm{R}^{+}$by translates of $\mathscr{T}$ where the elements in $\mathscr{T}$ are $t_{0}<t_{1}<t_{2}<\cdots$. Let $\varphi(x)=\frac{1}{t_{1}-t_{0}}\left(x-t_{0}\right)$ and $t_{j}^{\prime}=\varphi\left(t_{j}\right)$. Then

$$
\varphi(\Omega) \oplus\left\{0,1, t_{2}^{\prime}, t_{3}^{\prime}, \ldots\right\}=\mathrm{R}^{+} .
$$

Hence $\varphi(\Omega)=[0,1]+B$ for some $B \subset \mathrm{Z}^{+}$with $0 \in B$.

\section{Proofs of Main Theorems}

To prove our main theorems we first introduce some notation. For any finite set $A \subset \mathrm{Z}$ we denote $f_{A}(\xi):=A\left(e^{i 2 \pi \xi}\right)$ where $A(z)$ is the characteristic (Laurent) polynomial of $A$. We will use $\mathscr{Z}_{A}$ to denote the set of zeros of $f_{A}$. For a subset $\Omega \subset \mathrm{R}$ with positive and finite measure we will use $\mathscr{Z}_{\Omega}$ to denote the set of zeros of $\widehat{\chi}_{\Omega}(\xi)$.

Observe that for any finite $A \subset Z, \xi \in \mathscr{Z}_{A}$ implies $\xi+m \in \mathscr{Z}_{A}$ for all $m \in \mathrm{Z}$. So $\mathscr{Z}_{A}=\mathrm{Z} \oplus X$ for some finite $X \subset \mathrm{R}$. If in addition $A$ is a direct summand of $Z_{n}^{+}$for some $n \in \mathrm{N}$, then $n \mathscr{Z}_{A} \subseteq \mathrm{Z}$.

Lemma 4.1. Let $A \subset \mathrm{Z}^{+}$be a direct summand of $\mathrm{Z}_{n}^{+}$for some $n \in \mathrm{N}$. Then there exists a direct summand $A^{*}$ of $Z_{n}^{+}$with the same cardinality such that

$$
A-A \subseteq n \mathscr{Z}_{A^{*}} \cup\{0\}, \quad A^{*}-A^{*} \subseteq n \mathscr{Z}_{A} \cup\{0\} .
$$

Proof. We procced by induction on $n$. For $n=1,2$ it is easy to check that the lemma holds. Assume that the lemma holds for all $n<k$, where $k \geq 3$. We show that it holds for $n=k$.

Case $1.1 \notin A$. Then $A=r A_{1}$ for some $r>1, r \mid k$ and direct summand $A_{1}$ of $Z_{\frac{k}{r}}^{+}$. By the hypothesis there exists a direct summand $A_{1}^{*}$ of $Z_{\frac{k}{r}}^{+}$such that (4.1) holds for $A_{1}, A_{1}^{*}$ and $n=k / r$. Now $f_{A}(\xi)=f_{A_{1}}(r \xi)$ yields $\mathscr{Z}_{A}=\frac{1}{r} \mathscr{Z}_{A_{1}}$. Set $A^{*}=A_{1}^{*}$. Clearly $A^{*}$ is a direct summand of $Z_{k}^{+}$because it is a direct summand of $Z_{\frac{k}{r}}^{+}$, and we have

$$
A-A=r\left(A_{1}-A_{1}\right) \subseteq r \cdot \frac{k}{r} \mathscr{Z}_{A_{1}^{*}} \cup\{0\}=k \mathscr{Z}_{A^{*}} \cup\{0\},
$$

and

$$
A^{*}-A^{*}=A_{1}^{*}-A_{1}^{*} \subseteq \frac{k}{r} \mathscr{Z}_{A_{1}} \cup\{0\}=k \mathscr{Z}_{A} \cup\{0\} .
$$


Case 2. $1 \in A$. Then $A=Z_{r}^{+} \oplus r A_{1}$ for some $r>1, r \mid k$ and direct summand $A_{1}$ of $Z_{\frac{k}{r}}^{+}$. By the hypothesis there exists a direct summand $A_{1}^{*}$ of $Z_{\frac{k}{r}}^{+}$ such that (4.1) holds for $A_{1}, A_{1}^{*}$ and $n=k / r$. Set $A^{*}=A_{1}^{*} \oplus \frac{k}{r} Z_{r}^{+}$. $A^{*}$ is a direct summand of $\mathrm{Z}_{k}^{+}$because $A^{*} \oplus B_{1}^{*}=\mathrm{Z}_{k}^{+}$where $A_{1}^{*} \oplus B_{1}^{*}=\mathrm{Z}_{\frac{k}{r}}$. We have

$$
f_{A}(\xi)=f_{\mathrm{Z}_{r}^{+}}(\xi) f_{A_{1}}(r \xi), \quad f_{A^{*}}(\xi)=f_{A_{1}^{*}}(\xi) f_{\mathrm{Z}_{r}^{+}}\left(\frac{k}{r} \xi\right)
$$

It follows from $\mathscr{Z}_{\mathrm{Z}_{r}^{+}}=\frac{1}{r} \mathrm{Z} \backslash \mathrm{Z}$ that

$$
\mathscr{Z}_{A}=\frac{1}{r}\left(\mathrm{Z} \cup \mathscr{Z}_{A_{1}}\right) \backslash \mathrm{Z}, \quad \mathscr{Z}_{A^{*}}=\mathscr{Z}_{A_{1}^{*}} \cup \frac{r}{k}\left(\frac{1}{r} \mathrm{Z} \backslash \mathrm{Z}\right) .
$$

Let $m=a+\frac{k}{r} j$ and $m=a^{\prime}+\frac{k}{r} j^{\prime}$ be two distinct elements in $A^{*}$, where $a, a^{\prime} \in A_{1}^{*}$ and $j, j^{\prime} \in \mathrm{Z}_{r}^{+}$. If $a=a^{\prime}$ then

$$
m-m^{\prime}=\frac{k}{r}\left(j-j^{\prime}\right) \in k\left(\frac{1}{r} \mathrm{Z} \backslash \mathrm{Z}\right) \subseteq k \mathscr{Z}_{A} .
$$

If $a \neq a^{\prime}$ then $a-a^{\prime} \in \frac{k}{r} \mathscr{Z}_{A_{1}}$. Hence $a-a^{\prime}+\frac{k}{r} l \in \frac{k}{r} \mathscr{Z}_{A_{1}}$ for all $l \in$ Z. Since $m-m^{\prime} \notin k Z$, we have

$$
m-m^{\prime} \in \frac{k}{r} \mathscr{Z}_{A_{1}} \backslash k Z \subseteq k \mathscr{Z}_{A} .
$$

Hence $A^{*}-A^{*} \subseteq k \mathscr{Z}_{A} \cup\{0\}$.

Now let $m=j+r a, m^{\prime}=j^{\prime}+r a^{\prime}$ be two distinct elements in $A$, where $a, a^{\prime} \in A_{1}$ and $j, j^{\prime} \in Z_{r}^{+}$. If $j=j^{\prime}$ then $a \neq a^{\prime}$, and by the hypothesis $a-a^{\prime} \in \frac{k}{r} \mathscr{Z}_{A_{1}^{*}}$. So $m-m^{\prime}=r\left(a-a^{\prime}\right) \in k \mathscr{Z}_{A_{1}^{*}}$. If $j \neq j^{\prime}$ then $j-j^{\prime} \notin r \mathrm{Z}$, so

$$
m-m^{\prime}=j-j^{\prime}+r\left(a-a^{\prime}\right) \in \mathrm{Z} \backslash r \mathrm{Z}=\frac{r}{k}\left(\frac{1}{r} \mathrm{Z} \backslash \mathrm{Z}\right) \subseteq \mathscr{Z}_{A^{*}}
$$

Hence $A-A \subseteq \mathscr{Z}_{A^{*}}$.

We have now completed the induction steps and proven the lemma.

We will call two direct summand $A$ and $A^{*}$ satisfying (4.1) a conjugate pair, and $A^{*}$ a conjugate of $A$. The proof of Lemma 4.1 leads to an explicit construction of conjugate pairs. Let $A \subset \mathrm{Z}^{+}$be a direct summand of $\mathrm{Z}_{n}^{+}$. Then by Corollary 2.3 there exists a unique sequence $r_{0}, r_{1}, \ldots, r_{2 k+1}$ in $\mathrm{N}$ with $\prod_{j=0}^{2 k+1} r_{j}=n, r_{j}>1$ for $0<j<2 k+1$ and $r_{0}, r_{2 k+1} \geq 1$, such that

$$
A=\bigoplus_{j=0}^{k} d_{2 j} \mathrm{Z}_{r_{2 j+1}}^{+}, \quad \text { where } \quad d_{m}:=\prod_{j=0}^{m} r_{j}
$$


Define the map $\vartheta_{n}$ on the set of direct summand of $Z_{n}^{+}$by

$$
\vartheta_{n}(A)=\bigoplus_{j=0}^{k} \frac{n}{d_{2 j+1}} \mathrm{Z}_{r_{2 j+1}}^{+} .
$$

Then $\vartheta_{n}(A)$ is exactly the conjugate set $A^{*}$ constructed inductively in the proof of Lemma 4.1.

Lemma 4.2. Suppose that $A \subset \mathrm{Z}^{+}$is a direct summand of $\mathrm{Z}_{n}^{+}$. Then $A$ and $\vartheta_{n}(A)$ form a conjugate pair, and $\vartheta_{n}\left(\vartheta_{n}(A)\right)=$ A. Furthermore, if $A, B \subset \mathrm{Z}^{+}$ such that $A \oplus B=Z_{n}^{+}$, then $\vartheta_{n}(A) \oplus \vartheta_{n}(B)=Z_{n}^{+}$

Proof. The proof of Lemma 4.1 already implies that $A, \vartheta_{n}(A)$ form an conjugate pair. It is easy to see that $\vartheta_{n}\left(\vartheta_{n}(A)\right)=A$ by directly applying (4.3) and (4.4). Now, suppose that $A$ is given by (4.3) and $B \subset Z^{+}$satisfies $A \oplus B=Z_{n}^{+}$. Then there are several cases: $r_{0}=1$ or $r_{0}>1$, and $r_{2 k+1}=1$ or $r_{2 k+1}>1$. If $r_{0}=1, r_{2 k+1}>1$ then

$$
B=\bigoplus_{j=1}^{k+1} d_{2 j-1} \mathrm{Z}_{r_{2 j}}^{+}, \quad \text { where } \quad r_{2 k+2}:=1 .
$$

So

$$
\vartheta_{n}(B)=\bigoplus_{j=1}^{k+1} \frac{n}{d_{2 j}} \mathrm{Z}_{r_{2 j}}^{+} .
$$

It is now straightforward to check from (4.4) and (4.6) that $\vartheta_{n}(A) \oplus \vartheta_{n}(B)=$ $\mathrm{Z}_{n}^{+}$. Other cases can be checked similarly.

Definition 4.3. Let $\Lambda, \mathscr{T} \subset \mathrm{R}$ be strongly periodic sets. We say that $\mathscr{T}$ is a dual of $\Lambda$ if there exist a non-zero $\alpha \in \mathrm{R}$ and $A, B \subset \mathrm{Z}^{+}$with $A \oplus B=\mathrm{Z}_{n}^{+}$ for some $n \in \mathrm{N}$ such that

$$
\Lambda=\alpha(A \oplus n Z), \quad \mathscr{T}=\frac{1}{n \alpha}\left(\vartheta_{n}(B) \oplus n Z\right) .
$$

By Lemma 4.2 if $\mathscr{T}$ is a dual of $\Lambda$ then $\Lambda$ is a dual of $\mathscr{T}$.

Lemma 4.4. Let $\Omega \subset \mathrm{R}$ satisfy $\mu(\Omega)=n \in \mathrm{N}$. Suppose that $\Lambda=L \oplus \mathrm{Z}$ where $L$ is a finite subset of $\mathrm{R}$ such that $\Lambda-\Lambda \subseteq \mathscr{Z}_{\Omega} \cup\{0\}$. Then $(\Omega, \Lambda)$ is a spectral pair if and only if $|L|=n$.

Proof. See [10], Theorem 1, or [8], Theorem 2.1. 
We shall establish the following result, which is a stronger version of our main theorem.

THEOREM 4.5. Suppose that $\Omega \subset \mathrm{R}$ has positive and finite Lebesgue measure. Let $\Lambda, \mathscr{T} \subset \mathrm{R}$ be strongly periodic sets such that $\mathscr{T}$ is a dual of $\Lambda$. Then $(\Omega, \Lambda)$ is a spectral pair if and only if $\Omega$ tiles $R$ by translates of $\mathscr{T}$.

Proof. Without loss of generality we may assume that $\Lambda=\frac{1}{n}(A \oplus n Z)$ and $\mathscr{T}=\vartheta_{n}(B) \oplus n Z$ for some $n \in \mathrm{N}$ and $A, B \subset \mathrm{Z}^{+}$with $A \oplus B=\mathrm{Z}_{n}^{+}$.

$(\Leftarrow) \quad$ The set $\Omega^{\prime}=\Omega \oplus \vartheta_{n}(B)$ tiles R by translates of $n Z$, so it is a fundamental domain of the lattice $n Z$. Hence

$$
\mathscr{Z}_{\Omega^{\prime}}=\mathscr{Z}_{\Omega} \cup \mathscr{Z}_{\vartheta_{n}(B)} \supseteq \frac{1}{n} \mathrm{Z} \backslash\{0\} .
$$

Since $\vartheta_{n}(A) \oplus \vartheta_{n}(B)=\mathrm{Z}_{n}^{+}$we have

$$
\mathscr{Z}_{\vartheta_{n}(A)} \cup \mathscr{Z}_{\vartheta_{n}(B)}=\mathscr{Z}_{Z_{n}^{+}}=\frac{1}{n} \mathrm{Z} \backslash \mathrm{Z} .
$$

Furthermore, $\mathscr{Z}_{\vartheta_{n}(A)} \cap \mathscr{Z}_{\vartheta_{n}(B)}=\emptyset$ because $f_{\vartheta_{n}(A)}(\xi) f_{\vartheta_{n}(B)}(\xi)$ has no multiple roots. Hence

$$
\mathscr{Z}_{\Omega} \supseteq \mathscr{Z}_{\vartheta_{n}(A)} \cup Z \backslash\{0\} .
$$

Now, for any distinct $\lambda, \lambda^{\prime} \in \Lambda$ we have $\lambda-\lambda^{\prime}=\frac{1}{n} k+j$ for some $k \in A-A$, $j \in Z$. If $k \neq 0$ then $\frac{k}{n} \in \mathscr{Z}_{\vartheta_{n}(A)}$ by (4.1), which implies that $\lambda-\lambda^{\prime}=\frac{k}{n}+j \in$ $\mathscr{Z}_{\vartheta_{n}(A)} \subseteq \mathscr{Z}_{\Omega}$. Otherwise $\lambda-\lambda^{\prime}=j \in Z \backslash\{0\} \subseteq \mathscr{Z}_{\Omega}$. By Lemma $4.4(\Omega, \Lambda)$ is a spectral pair.

$(\Rightarrow)$ Suppose that $(\Omega, \Lambda)$ is a spectral pair. For any $x \in[0,1)$ let $D_{x}:=$ $\Omega \cap(Z+x)$. It follows from [10], Theorem 2 , that

$$
\left|D_{x}\right|=|A|, \quad D_{x}-D_{x} \subseteq n \mathscr{Z}_{A} \cup\{0\}
$$

for almost all $x \in[0,1)$. We show that $\left(D_{x}-x\right)+\vartheta_{n}(B)$ is a complete residue system $(\bmod n)$ for every $D_{x}$ satisfying (4.7). Note that $\vartheta_{n}(B)-\vartheta_{n}(B) \subseteq$ $n \mathscr{Z}_{B} \cup\{0\}$, and observe that $k \not \equiv m \bmod n$ for any $k \in n \mathscr{Z}_{A}$ and $m \in n \mathscr{Z}_{B}$. Thus for any $k_{1}, k_{2} \in D_{x}-x$ and $m_{1}, m_{2} \in \vartheta_{n}(B)$ we must have $k_{1}-k_{2} \not \equiv$ $m_{2}-m_{1} \bmod n$ unless $k_{1}=k_{2}$ and $m_{1}=m_{2}$. Hence $k_{1}+m_{1} \not \equiv k_{2}+m_{2} \bmod n$. Since $\left|D_{x}-x\right| \cdot\left|\vartheta_{n}(B)\right|=n$ it follows that $\left(D_{x}-x\right)+\vartheta_{n}(B)=\left(D_{x}-x\right) \oplus$ $\vartheta_{n}(B)$ contains $n$ distinct residue classesmod $n$, and hence is a complete residue systemmod $n$. Therefore

$$
D_{x}+\mathscr{T}=D_{x} \oplus \mathscr{T}=x+\mathrm{Z}
$$

for almost all $x \in[0,1)$. This implies that $\Omega$ tiles $\mathrm{R}$ by translates of $\mathscr{T}$. 
Theorem 1.1 is a simple consequence of Theorem 3.1 and Theorem 4.5.

Acknowledgement. The first author would like to thank Palle E. T. Jorgensen for many useful conversations about spectral pairs.

\section{REFERENCES}

1. Carlitz and Moser, On some special factorizations of $\left(1-x^{n}\right) /(1-x)$, Canad. Math. Bull. 9 (1966), 421-426.

2. de Bruijn, N. G., On number systems, Nieuw Arch. Wisk. (4) 4 (1956), 15-17.

3. Fuglede, B., Commuting self-adjoint partial differential operators and a group theoretic problem, J. Funct. Anal. 16 (1974), 101-121.

4. Jorgensen, P. E. T., and Pedersen, S., Spectral theory for Borel sets in $\mathrm{R}^{n}$ of finite measure, J. Funct. Anal. 107 (1992), 72-104.

5. Jorgensen, P. E. T., and Pedersen, S., Harmonic analysis and fractal limit-measures induced by representations of a certain $C^{*}$-algebra, J. Funct. Anal. 125 (1994), 90-110.

6. Jorgensen, P. E. T., and Pedersen, S., Orthogonal harmonic analysis of fractal measures, Electron. Res. Announc. Amer. Math. Soc. 4 (1998), 35-42.

7. Lagarias, J. C. and Wang, Y., Tiling the line with translates of one tile, Invent. Math. 124 (1996), 341-365.

8. Lagarias, J. C. and Wang, Y., Spectral sets and factorizations of finite abelian groups, J. Funct. Anal. 145 (1997), 73-98.

9. Odlyzko, A. M., Non-negative digit sets in positional number systems, Proc. London Math. Soc. 37 (1978), 213-229.

10. Pedersen, S., Spectral sets whose spectrum is a lattice with a base, J. Funct. Anal. 141 (1996), 496-509.

STEEN PEDERSEN

DEPARTMENT OF MATHEMATICS

WRIGHT STATE UNIVERSITY

DAYTON OH 45435

USA

E-mail: steen@math.wright.edu
YANG WANG

DEPARTMENT OF MATHEMATICS

GEORGIA INSTITUTE OF TECHNOLOGY

ATLANTA GA 30332

USA

E-mail: wang@math.gatech.edu 